\title{
Bundesweites Erfassungssystem für Lebensmittel, die bei Ausbrüchen beteiligt sind
}

\author{
Dr. P. Hiller, Dr. A. Gervelmeyer und Dr. J. Bräunig \\ Bundesinstitut für Risikobewertung (BfR), Diedersdorfer Weg 1, 12277 Berlin
}

Lebensmittelbedingte Infektionen und Intoxikationen des Menschen können von einer Vielzahl bakterieller, viraler und parasitologischer Erreger bzw. durch sie gebildete Toxine verursacht werden. In der Mehrzahl führen sie zu Gastroenteritiden, die oft einen milden, selbstlimitierenden Verlauf nehmen; sie können aber auch schwere, mitunter lebensbedrohliche Syndrome verursachen.

Für die rasche Kontrolle eines aktuellen Ausbruchsgeschehens mit dem Ziel einer Vermeidung weiterer humaner Erkrankungsfälleistes notwendig, dass dieLebensmittelüberwachungsund Gesundheitsbehörden auf der Ebene der betroffenen Kreise kontinuierlich eng zusammenarbeiten und frühzeitig relevante Informationen austauschen. Um lebensmittelbedingte Krankheitsausbrüche zu verhindern sind Kenntnisse über die involvierten Erreger sowie deren Ursprung dringend erforderlich.

Die Europäische Kommission hat daher in ihrer Richtlinie 2003/99/EG zur Überwachung von Zoonosen und Zoonoseerregern die Aufklärung von lebensmittelbedingten Ausbrüchen und die regelmäßige Übermittlung der daraus resultierenden Daten festgeschrieben.

In Deutschland wurde bereits im Jahr 1982 ein nationales Meldesystem zur Zentralen Erfassung Von Ausbrüchen Lebensmittelbedingter Infektionen und Intoxikationen (ZEVALI) eingeführt. In einer Pilotphase im Jahr 2003 wurde die Anwendung eines überarbeiteten Systems, in je 2 Kreisen aus fünf Ländern sowie im Regierungsbezirk Schwaben, getestet und anschließend beschlossen, es bundesweit einzuführen. Dieses Erfassungssystem für Lebensmittel wird vom BfR geführt und dient der zentralen Sammlung von Daten zu den Ursachen und den epidemiologischen Zusammenhängen bei Lebensmittelinfektionen und -intoxikationen. Es wurden drei Fragebögen zu den beteiligten Lebensmitteln (Mantelbogen, LebensmittelErfassungsbogen und Proben-Erfassungsbogen) entwickelt, die von den an der Aufklärung von Ausbrüchen beteiligten Behörden auszufüllen sind ${ }^{1}$.

Zur Erläuterung der Fragebögen steht ein ausführliches Handbuch unter der genannten Adresse zur Verfügung. Die Fachgruppe ,Aufklärung von Ausbrüchen` des BfR bietet allen interessierten Anwendern individuelle Hilfe per Telefon oder Email sowie Einführungsveranstaltungen und Workshops an.
Weiterhin können auch interdisziplinäre Kurse zum Vorgehen bei der Ausbruchsaufklärung in Zusammenarbeit mit dem Robert Koch-Institut durchgeführt werden.

Die Daten werden vom BfR in einer Datenbank erfasst und analysiert. Die erstellten Berichte werden an alle Beteiligten sowie weitere relevante Institutionen verteilt und stellen die Grundlage für die Erfüllung der Berichtspflicht an die EFSA gemäß der EU-Zoonosen-Überwachungs-RL 2003/99 dar. Die Ergebnisse sollen außerdem für eine qualitative und quantitative Risikobewertung für verschiedene Erreger-Lebensmittel-Kombinationen genutzt werden.

Die nachfolgende Grafik zeigt die einzuleitenden Verfahrensschritte beim Verdacht auf einen lebensmittelbedingten Ausbruch:

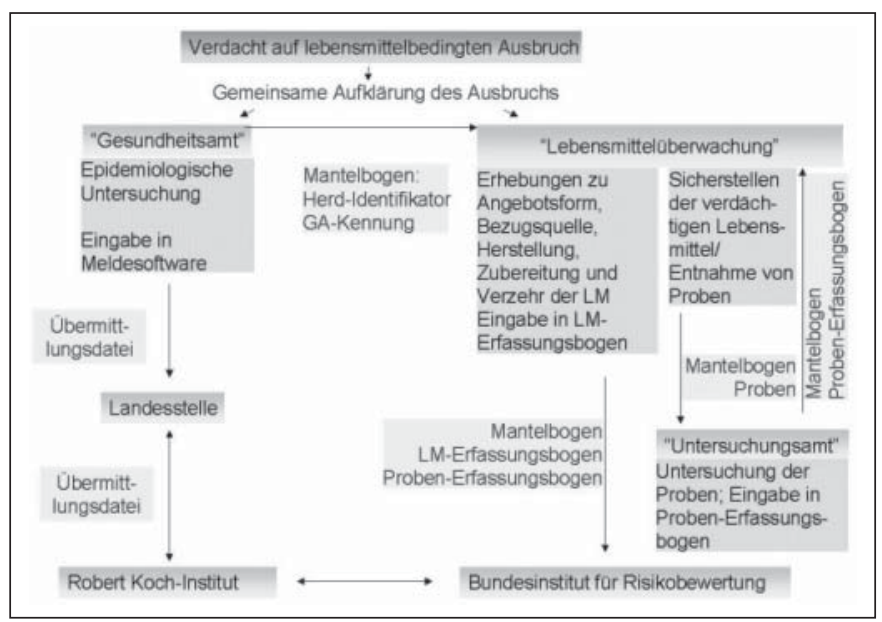

\section{Beschluss}

Kenntnisnahme

${ }^{1}$ Sie sind auf der BfR Internetseite unter Biologische Sicherheit-Mikrobielle Risiken - Erfassung lebensmittelbedingter Ausbrüche als zip-Datei abrufbar und können per Email, Fax oder Brief an das BfR unter Bundesinstitut für Risikobewertung, Fachgruppe Aufklärung von Ausbrüchen, Diedersdorfer Weg 1, 12277 Berlin, Tel. 030/ 8412-2140 oder -2158, Fax 030/8412-2951, Email: zevali@bfr.bund.de zurückgeschickt werden. 